\title{
Implementation of multidrug-resistant tuberculosis (MDR-TB) treatment in Gabon: lessons learnt from the field
}

\author{
U. Ateba-Ngoa ${ }^{1,3}$ - J. R. Edoa ${ }^{1,3}$ - B. R. Adegbite, ${ }^{1,3} \cdot$ E. G. Rossatanga ${ }^{2} \cdot$ D. Madiou ${ }^{2} \cdot$ A. Mfoumbi $^{1} \cdot$ C. Mevyann ${ }^{1}$. \\ P. Achimi Agbo ${ }^{1}$. J. Mahoumbou ${ }^{4}$. S. Gould ${ }^{1}$ - B. Lell ${ }^{1,3} \cdot$ A. A. Adegnika ${ }^{1,3} \cdot$ C. Köhler ${ }^{1,3} \cdot$ P. G. Kremsner ${ }^{1,3}$. \\ M. Massinga-Loembe ${ }^{1,3} \cdot$ A. Alabi ${ }^{1,3} \cdot$ M. P. Grobusch ${ }^{1,3,5}$
}

Received: 30 November 2018 / Accepted: 30 April 2019 / Published online: 9 May 2019

(c) The Author(s) 2019

\begin{abstract}
Purpose Since May 2016, WHO recommended a 9-12 month short-treatment regimen for multidrug-resistant tuberculosis (MDR-TB) treatment known as the 'Bangladesh Regimen'. However, limited data exist on the appropriateness thereof, and its implementation in low- and middle-income countries (LMIC). We report here on the pilot phase of the evaluation of the Bangladesh regimen in Gabon, prior to its endorsement by the WHO.

Methods This ongoing observational study started in September 2015. Intensive training of hospital health workers as well as community information and education were conducted. GeneXpert-confirmed MDR-TB patients received the secondline anti-tuberculosis drugs (4KmMfxPtoHCfzEZ/5MfxCfzEZ). Sputum smears and cultures were done monthly. Adverse events were monitored daily.

Results Eleven patients have been treated for MDR-TB piloting the short regimen. All were HIV-negative and presented in poor health with extensive pulmonary lesions. The overall sputum culture conversion rate was $64 \%$ after 4 months of treatment. Three patients developed marked hearing loss; one a transient cutaneous rash. Of 11 patients in our continuous care, 7 (63.6\%) significantly improved clinically and bacteriologically. One (9.1\%) patient experienced a treatment failure, two $(18.2 \%)$ died, and one (9.1\%) was lost to follow up.

Conclusions Our pioneering data on systematic MDR-TB treatment in Gabon, with currently almost total absence of resistance against the second-line drugs, demonstrate that a 9-month regimen has the capacity to facilitate early culture negativity and sustained clinical improvement. Close adverse events monitoring and continuous care are vital to success.
\end{abstract}

Keywords Multidrug resistance $\cdot$ Tuberculosis $\cdot$ Gabon $\cdot$ Short-course regimen

\section{Introduction}

U. Ateba-Ngoa, J. R. Edoa, and B. R. Adegbite have contributed equally.

Electronic supplementary material The online version of this article (https://doi.org/10.1007/s15010-019-01314-5) contains supplementary material, which is available to authorized users.

M. P. Grobusch

m.p.grobusch@amsterdamumc.nl

1 Centre des Recherches Médicales de Lambaréné, Lambarene, Gabon

2 Centre Hospitalier Régional Georges Rawiri de Lambaréné, Lambarene, Gabon

3 Institut für Tropenmedizin, Eberhard Karls Universität, Tübingen, Germany
Multidrug-resistant tuberculosis (MDR-TB) and higher degrees of resistant tuberculosis are an emerging health concern including populations in sub-Saharan Africa, and a massive threat to TB programmes in many low- and middle-income countries [1]. However, in the majority of these

4 Programme National de Lutte contre la Tuberculose, Ministry of Health, Libreville, Gabon

5 Department of Infectious Diseases, Center of Tropical Medicine and Travel Medicine, Amsterdam University Medical Centers, Location AMC, University of Amsterdam, Amsterdam, The Netherlands 
countries, patients often only have very limited access to MDR-TB treatment and data are still scarce on incidence, prevalence, molecular characteristics, treatment, and outcome [1]. In Gabon, despite case reports of treatment failure to the first-line TB therapy [2], it was only in 2013 that a more comprehensive assessment of the epidemiology of MDR-TB was conducted for the first time [3]. This study, carried out in Lambaréné, a semi-urban town of around 30.000 inhabitants, indicated an MDR rate of 4/91 (4\%) and $4 / 13(31 \%)$ in new and retreatment TB cases, respectively [3]. This rate is quite high and is comparable to prevalences reported from highly MDR-TB endemic countries [4, 5]. At the time of the official report on the first MDR-TB cases in Lambaréné and their growing number, the Gabonese TB programme lacked access to the second-line anti-tuberculosis drugs as well as specialized and dedicated treatment facilities [6]. In response to this epidemic, MDR-TB was recognized as a public health emergency in Gabon; and in September 2015, the first MDR-TB treatment center was opened in Lambaréné, at the Georges Rawiri Hospital (GRH), with technical support provided by CERMEL's clinical and mycobacteriology laboratory teams. In parallel, the Centre de Recherches Médicales de Lambaréné (CERMEL) obtained funding from the German Ministry of Health for the acquisition of the second-line anti-tuberculosis drugs for the evaluation of a 9-12 month short-course treatment regimen of MDR-TB. This short-course treatment was already evaluated by the International Union Against Tuberculosis and Lung Disease (IUALTD, 'The Union') in several countries in Africa and in Bangladesh prior to its endorsement by the WHO in May 2016 [7]. As a result of the collaboration between CERMEL and the Ministry of Health in Gabon, the first MDR-TB patients were initiated on a short-course treatment regimen in September 2015; in a study aimed at evaluating the implementation of the 9-12 month short regimen for the treatment of MDR-TB. We report here the experience gained during the treatment of those first MDR-TB patients in Gabon' and comment on the appropriateness of the standard 9-month regimen in our setting.

\section{Methods}

\section{Study design}

Data presented in this small pilot case series were collected from the first participants—with bacteriologically confirmed multidrug-resistant tuberculosis (MDR-TB). The study protocol was largely inspired by The Union research protocol implemented in nine countries in Africa, aimed at testing the efficacy and effectiveness of a 9-month short-course regimen for the treatment of MDR-TB [8].

\section{Study site and study population}

This ongoing study is conducted by CERMEL. The patients, all originating from the Moyen Ogooue province of Gabon, were followed up at the GRH in Lambaréné and at the Bongolo Evangelical Hospital (BH) in Lebamba. The study population consists of consecutive patients with bacteriologically confirmed MDR-TB, and no randomisation procedure was performed. Patients were included in the study if they are 15 years of age or older; have never been treated with the second-line anti-TB drugs for more than 1 month; and provided written informed consent to participate and intend to adhere to treatment, including hospitalisation for possibly months according to need, and willingness to accept treatment directly observed by a health worker. Patients were excluded from the study in case of pregnancy; a very poor clinical condition as judged by the specialist clinician in charge; a history of hypersensitivity to any of the drugs or a QTc interval of longer than $500 \mathrm{~ms}$ in the baseline ECG.

\section{MDR-TB diagnosis}

Samples for mycobacterial investigation were first analysed by microscopy as per the national guidelines. Both the conventional Ziehl-Neelsen ( $\mathrm{ZN})$ and auramine fluorochrome (FM) staining were used, with interpretation (positivity) based on the results of the latter. Samples were concomitantly processed with the Gene Xpert MTB/ RIF assay according to the manufacturer's instructions. The mycobacteria growth indicator tube, MGIT (BACTEC MGIT 960 TB System, BD Diagnostics), was used for mycobacterial culture and drug susceptibility testing (DST). Mycobacterium tuberculosis Complex (MTBC) was confirmed in positive cultures by the GenoType MTBC assay (Hain Life Sciences, Nehren, Germany). DST of confirmed MTBC isolates to the first-line drugs RIF, INH, EMB, PZA, and STR was performed using the MGIT 960 system in accordance with the manufacturer's instructions (BBLTM MGITTM SIRE and PZA test kits, Becton-Dickinson, Franklin Lakes, NJ, USA). In case of any resistance to the first-line drugs, DST was also performed for the following second-line drugs: ethionamide (ETO), ofloxacin (OFX), cycloserine (CS), amikacin (AM), 4-aminosalicylic acid (PAS), and capreomycin (CM). For quality control purposes and genotyping, part of the samples was referred to a collaborating supranational laboratory, the German National Reference Center (NRC) for Mycobacteria in Borstel, Germany. 


\section{Treatment regimen}

The treatment regimen comprises of an intensive phase of 4 months with Kanamycin (Km), Moxifloxacin (Mfx), Prothionamide (Pto), Clofazimine (Cfx), Isoniazid (H), Ethambutol (E), and Pyrazinamide (Z) (4KmMfxPtoHCfzEZ) followed by a continuation phase of 5 months comprising MfxCfzEZ. All drugs are given daily under direct observation (DOT); dosage of each medication is according to weight.

\section{Follow-up during treatment}

All patients were hospitalised during the intensive phase to increase treatment adherence and for optimal recording of drug-related adverse events. During this phase, direct sputum smear microscopy and culture were performed monthly to monitor sputum conversion. If direct sputum smear microscopy and sputum culture remained positive at the end of month 4 , the intensive phase was extended for a maximum of 2 months [8]. Treatment of patients failing to respond bacteriologically (lack of conversion) at the end of month 6 would be discontinued and switched to individualised treatment, subject to availability of appropriate replacement drugs. Patients who had sputum conversion at the end of intensive phase were discharged from hospital at the start of the continuation phase. Subsequently, treatment was provided daily at home under direct observation by a trained nurse, who also records any possible drugrelated adverse events. All patients undergo periodic clinical and laboratory check-ups. To document any relapse, patients were followed up actively for 6 months, and were allowed to re-visit the research centre or the GRH unrestrictedly and for an unlimited period after the treatment period.

\section{Monitoring and management of adverse drugs events}

Adverse events, or absence thereof, were recorded daily by an attending nurse or physician during the medical round. Monitoring for adverse drug events also includes clinical evaluations and laboratory investigations performed periodically.

\section{Data management and statistical analysis}

Data on medical file were captured using Excel 2010. Treatment outcome was determined according to WHO definitions and its last reporting framework for tuberculosis [9].
Per-protocol and intention-to-treat analyses were applied to characterize treatment outcome.

\section{Ethical considerations}

The study protocol was explained to each patient. Written informed consent was obtained from each participant or their legal representative before inclusion. The study was approved by the Gabon National Ethics Committee "Comité National d'Éthique pour la Recherche" (CNER) and received authorisation from the Ministry of Public Health of Gabon.

\section{Results}

\section{Patient description}

The study started on 15th September 2016. We present data collected from the first 11 MDR-TB patients who have been treated with the 9-month short regimen. Patient characteristics on admission are presented in Table 1. In summary, age ranged from 19 to 62 years with a median of 27 years. The sex ratio (M:F) was 1.75. All patients were HIV-negative; one was diabetic (diagnosed and under treatment before MDR-TB treatment initiation). Eight patients were previously treated for DS-TB, with most of them having received at least two treatment courses. The median number of previous DS-TB treatment episodes per patient was 3.

Timing of routine examinations and laboratory investigations are summarized in Supplementary Table 1. Sputum examination at baseline showed the presence of AFB in all patients. Resistance to rifampicin was established by Gene Xpert MTB/RIF testing and further confirmed phenotypically by culture and DST. All patients' isolates were resistant to rifampicin, isoniazid, and pyrazinamide.

\section{Treatment outcomes}

Seven patients $(63.6 \%)$ were cured, one $(9.1 \%)$ failed treatment, two $(18.2 \%)$ died, and one $(9.1 \%)$ was lost to follow up and could not be evaluated [9]. The overall sputum culture conversion rate was $64 \%$ after 4 months of treatment. All cured patients were actively followed up for additional 6 months after treatment, and none experienced a relapse.

\section{Adverse events}

In general, all patients developed at least one AE. Gastrointestinal symptoms were the most frequent, but usually diminished by the end of the intensive phase. Five patients presented signs of a decreased hearing capacity that could not be objectified/quantified due to lack of audiometric testing equipment. At month 2, one patient presented with a 


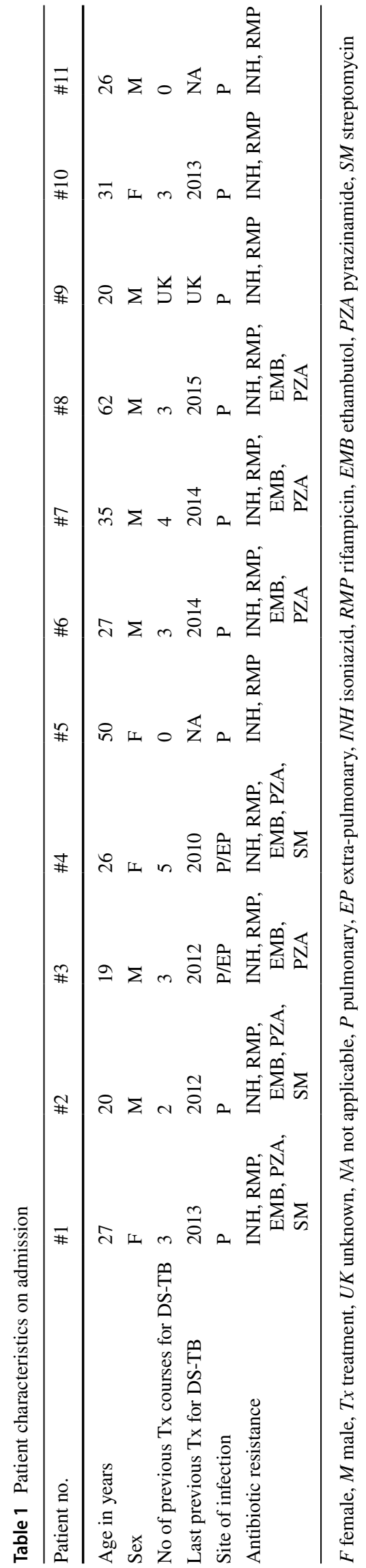

serious cutaneous hypersensitivity reaction. This condition resolved at month 4 after adequate treatment. One patient presented with massive haemoptysis and one other patient transferred back at her own request to another hospital beyond reach for our team which developed terminal hepatic failure about 4 months after treatment initiation. Supplementary Table 2 provides the details of the nature and occurrence of adverse events (AEs) during the treatment phase and their duration.

\section{Cost of treatment}

The total cost of the treatment of all patients reported here amounted to 61,432 USD. The mean cost of the MDR-TB treatment per patient was 5585 USD. This overall cost included hospitalisation, laboratory tests, nutritional support, and drug costs; with the majority of drugs having been sourced through donations, the hospitalisation costs amounted to $80.6 \%$ of the total cost of treatment.

\section{Discussion}

MDR-TB represents an emerging public health concern in Gabon. Despite the increasing number of cases, few, if any, treatment options were available in the country until recently $[2,3,10]$. Here, we report on the outcome of a pilot treatment phase that aimed to introduce and implement the 9-12 month short-regimen scheme before its endorsement by the WHO in May 2016 [7].

The appropriateness of the abridged WHO MDR-TB regime has been disputed [11]. Lange et al. [11] point out that in view of the respective prevailing drug resistance patterns e.g., in the Western European region and in the Eastern European region, only 7.8\% [12] and 4.2\% [13] of patients with DR-TB would be eligible for the short-course regimen. In Singapore, Indonesia and other South East Asian countries, the figure would be $30 \%$ [14]; of the other regions examined, only Brazil would yield 50-55\% eligible patients [15]. However, with until recently an almost complete absence of second- and third-line TB drugs [3], our setting renders patient cohorts such as the one presented here as an ideal candidate for the abridged WHO regimen, which is in situations where due to previous lack of access to second-line treatments, resistance to second-line drugs is by-and-large absent.

The medical history of the patients included in this case series clearly indicates a delay in the diagnosis of MDR-TB that led to unnecessary and ineffective treatment with the first-line drugs. Consequently, this led to profound health deterioration, extensive lung lesions, and irreversible sequelae such as chronic respiratory failure. Moreover, DST indicated that mycobacteria isolated from 
all patients were resistant to at least three of the first-line anti-tuberculosis drugs, which could be a consequence of repeated treatment [16] emphasising the importance of early diagnosis and treatment of MDR-TB.

Under the standardized treatment regimen, sputum culture conversion was obtained already after 3 months of treatment in nine patients, whereas sputum conversion by microscopy, possibly due to the presence of dead bacteria, was observed after 4 months of treatment in seven patients. This confirms the necessity of culture for the monitoring of treatment outcome. A similar delay in smear conversion over culture conversion has been reported elsewhere [17] and is probably linked to the presence of dead bacilli hidden in cavities and later on released into the airways. Given that infectiousness is associated with positive sputum culture results and is highest when the smear results are also positive [18], data from our small patient cohort indicate that MDRTB patients' infectiousness was already reduced by month 2 and reached null by month 3 of treatment (data not shown).

All patients experienced at least one AE during the treatment phase that was managed accordingly. As expected, the most common AEs were gastro-intestinal and consisted mainly of nausea and vomiting. One patient experienced a cutaneous hypersensitivity reaction that was not clearly attributable to an individual drug. This reaction resolved after 2.5 months of symptomatic management without discontinuation of therapy. Towards the end of the continuation phase, two patients complained about a significant hearing loss. Although this is surely caused by the prolonged administration of kanamycin, it is possible that these patients who already received streptomycin several times before already had established sub-clinical cochlear lesions. Unfortunately, due to logistical constraints, we were not able to quantify the hearing loss. Management of MDR-TB patients is multidisciplinary and usually requires expertise and medical equipment not available in remote hospitals. This could affect the outcome of their treatment particularly with regard to the monitoring, prevention, and treatment of drug adverse effects. This limitation should be addressed in the future by encouraging the policy maker to provide appropriate logistic tool for efficient monitoring.

One patient died in the first month of treatment. Clinical and radiological features on admission depicted extensive lung damage that could be linked to delay in receiving adequate MDR-TB treatment. Liver failure occurred in one patient towards the end of the intensive phase. This patient who worked in Libreville was referred to our team in Lambaréné for the initiation of her MDR-TB treatment. Hospitalisation in Lambaréné was originally planned to last for the whole duration of the intensive phase. However, due to family and professional issues, the patient was discharged from our MDR-TB treatment unit and referred to a treating physician in Libreville. Liver enzymes before discharge in Lambaréné were within normal ranges.

The cost of treatment in our patient cohort is lower compared to a study in South Africa which reported 17,164 USD per patient [19], but our findings equate to those reported from, e.g., the Philippines, Peru, and Nigeria (3613; 2423, 2095 USD, respectively) [20, 21]. The treatment cost is strongly correlated with the country's socio-economic profile and the level of hospital care, as well as the sourcing/ financing of the drugs. However, all of these studies agree that the largest cost of care is attributable to the prolonged hospitalisation. Despite evidence that hospitalisation is desirable during the infective period and to achieve the highest rate of adherence to treatment, ambulatory care approaches should be encouraged where possible and as early as possible to reduce the cost of treatment.

In summary, the implementation of the MDR-TB shorttreatment regimen led to clinical improvement and sputum conversion in most of the patients. Early treatment could prevent extensive lung damage and death. All patients experienced at least one adverse event that included adverse cutaneous drug reaction and impaired hearing function, but did not lead to an interruption of the treatment. One patient experienced a severe and fatal adverse event. The problems of managing this patient highlight the importance of expanding the MDR-TB treatment capacity to other provinces to facilitate country-wide appropriate patient care. At the time of this study, Lambaréné was the only site of MDR-TB treatment. Patients could not be transferred safely to other province near to their residence to continue the treatment.

Acknowledgements We acknowledge all patients and site staff at CERMEL and Georges Rawiri Hospital. We thank Dr. Matthew McCall, CERMEL, for his thoughtful comments. We also wish to acknowledge the Damien Foundation who provided logistical and technical assistance for procurement of the second-line drugs.

Funding This work was supported by Deutsches Zentrum fuer Infektiologie (DZIF), the Federal Ministry of Education and Research (BMBF), and the EDCTP PANACEA I Grants.

\section{Compliance with ethical standards}

Conflict of interest None of the authors has a conflict of interest to declare.

Open Access This article is distributed under the terms of the Creative Commons Attribution 4.0 International License (http://creativeco mmons.org/licenses/by/4.0/), which permits unrestricted use, distribution, and reproduction in any medium, provided you give appropriate credit to the original author(s) and the source, provide a link to the Creative Commons license, and indicate if changes were made. 


\section{References}

1. Nourzad S, Jenkins HE, Milstein M, Mitnick CD. counting the missing cases: estimating the global burden of multidrug-resistant tuberculosis among prevalent cases of tb. Int $\mathrm{J}$ Tuberc Lung Dis Off J Int Union Tuberc Lung Dis. 2017;21:6-11.

2. Mounguengui D, Ondounda M, Lawson JMM, Fabre M, Gaudong L, Mangouka L, et al. Tuberculose multirésistante à l'hôpital d'instruction des armées de Libreville (Gabon) à propos de 16 cas. Bull Société Pathol Exot. 2012;105:1-4.

3. Bélard S, Remppis J, Bootsma S, Janssen S, Kombila DU, Beyeme $\mathrm{JO}$, et al. Tuberculosis treatment outcome and drug resistance in lambaréné, gabon: a prospective cohort study. Am J Trop Med Hyg. 2016;95:472-80.

4. Zignol M, Dean AS, Falzon D, van Gemert W, Wright A, van Deun A, et al. Twenty years of global surveillance of antituberculosis-drug resistance. N Engl J Med. 2016;375:1081-9.

5. Pablos-Méndez A, Raviglione MC, Laszlo A, Binkin N, Rieder HL, Bustreo F, et al. Global surveillance for antituberculosis-drug resistance, 1994-1997. World Health Organization-International Union against Tuberculosis and Lung Disease Working Group on Anti-Tuberculosis Drug Resistance Surveillance. N Engl J Med. 1998;338:1641-9.

6. Bélard S, Janssen S, Osbak KK, Adegnika AA, Ondounda M, Grobusch MP. Limited access to drugs for resistant tuberculosis: a call to action. J Public Health Oxf Engl. 2015;37:691-3.

7. WHO Treatment Guidelines for Drug-Resistant Tuberculosis, 2016 Update. Geneva: World Health Organization; 2016. http:// www.ncbi.nlm.nih.gov/books/NBK390455/. Accessed 17 Jun 2017.

8. Trébucq A, Schwoebel V, Kashongwe Z, Bakayoko A, Kuaban C, Noeske J, et al. Treatment outcome with a short multidrug-resistant tuberculosis regimen in nine African countries. Int $\mathbf{J}$ Tuberc Lung Dis. 2018;22:17-25. http://www.ingentaconnect.com/conte nt/10.5588/ijtld.17.0498. Accessed 18 Feb 2019.

9. WHOI Definitions and reporting framework for tuberculosis. WHO. http://www.who.int/tb/publications/definitions/en/. Accessed 28 Jul 2018

10. Alabi AS, Traoré AN, Loembe MM, Ateba-Ngoa U, Frank M, Adegnika AA, et al. Enhanced laboratory capacity development: a boost for effective tuberculosis control in resource-limited settings. Int J Infect Dis IJID Off Publ Int Soc Infect Dis. 2017;56:81-4.
11. Lange C, Chesov D, Heyckendorf J, Leung CC, Udwadia Z, Dheda K. Drug-resistant tuberculosis: an update on disease burden, diagnosis and treatment. Respirol Carlton Vic. 2018;23:656-73.

12. Lange C, Duarte R, Fréchet-Jachym M, Guenther G, Guglielmetti $\mathrm{L}$, Olaru ID, et al. Limited benefit of the new shorter multidrugresistant tuberculosis regimen in Europe. Am J Respir Crit Care Med. 2016;194:1029-31.

13. Balabanova Y, Fiebig L, Ignatyeva O, Riekstina V, Danilovits M, Jaama K, et al. Multidrug-resistant TB in Eastern region of the EU: is the shorter regimen an exception or a rule? Thorax. 2017;72:850-2.

14. Chee CBE, KhinMar KW, Sng LH, Jureen R, Cutter J, Lee VJM, et al. The shorter multidrug-resistant tuberculosis treatment regimen in Singapore: are patients from South-East Asia eligible? Eur Respir J. 2017. https://doi.org/10.1183/13993003.00753-2017.

15. Dalcolmo M, Gayoso R, Sotgiu G, D’Ambrosio L, Rocha JL, Borga L, et al. Effectiveness and safety of clofazimine in multidrug-resistant tuberculosis: a nationwide report from Brazil. Eur Respir J. 2017. https://doi.org/10.1183/13993003.02445-2016.

16. Berhan A, Berhan Y, Yizengaw D. A meta-analysis of drug resistant tuberculosis in sub-saharan africa: how strongly associated with previous treatment and HIV co-infection? Ethiop J Health Sci. 2013;23:271-82.

17. Ko Y, Shin JH, Lee H-K, Lee YS, Lee S-Y, Park SY, et al. Duration of pulmonary tuberculosis infectiousness under adequate therapy, as assessed using induced sputum samples. Tuberc Respir Dis. 2017;80:27-34.

18. Guidelines for the Investigation of contacts of persons with infectious tuberculosis $</ \mathrm{P}><\mathrm{P}>$ recommendations from the National Tuberculosis Controllers Association and CDC. https://www.cdc. gov/mmwr/preview/mmwrhtml/rr5415a1.htm. Accessed 17 Jun 2017.

19. Schnippel K, Rosen S, Shearer K, Martinson N, Long L, Sanne I, et al. Costs of inpatient treatment for multi-drug-resistant tuberculosis in South Africa. Trop Med Int Health TM IH. 2013;18:109-16.

20. Musa BM, John D, Habib AG, Kuznik A. Cost-optimization in the treatment of multidrug resistant tuberculosis in Nigeria. Trop Med Int Health. 21:176-82. https://onlinelibrary.wiley.com/doi/ abs/10.1111/tmi.12648. Accessed 28 Jul 2018.

21. Fitzpatrick C, Floyd K. A systematic review of the cost and cost effectiveness of treatment for multidrug-resistant tuberculosis. PharmacoEconomics. 2012;30:63-80. 